\title{
Natural Convection for Slip Flow in a Vertical Polygonal Duct With a Heated Core
}

\author{
Hang Yuan, Chiu-On $\mathrm{Ng}^{1}$ \\ Department of Mechanical Engineering, The University of Hong Kong, \\ Pokfulam Road, Hong Kong \\ C. Y. Wang \\ Department of Mathematics, Michigan State University, \\ East Lansing, MI 48824
}

April 8, 2015

\begin{abstract}
Free convection with velocity slip and temperature jump in a vertical polygonal duct with a heated circular core is semi-analytically solved by the methods of eigenfunction expansion and point match. The conditions of the core wall being at uniform wall temperature or uniform heat flux are considered. It is shown that the temperature and flow fields can be very different from those without the velocity slip and temperature jump. The optimum core radius and the corresponding maximum flow rate are numerically determined for some values of the slip length, temperature jump coefficient, and the number of sides of the polygon.
\end{abstract}

Keywords: natural convection; slip flow; temperature jump; polygonal duct.

\footnotetext{
${ }^{1}$ Corresponding author. E-mail: cong@hku.hk.
} 


\section{Nomenclature}

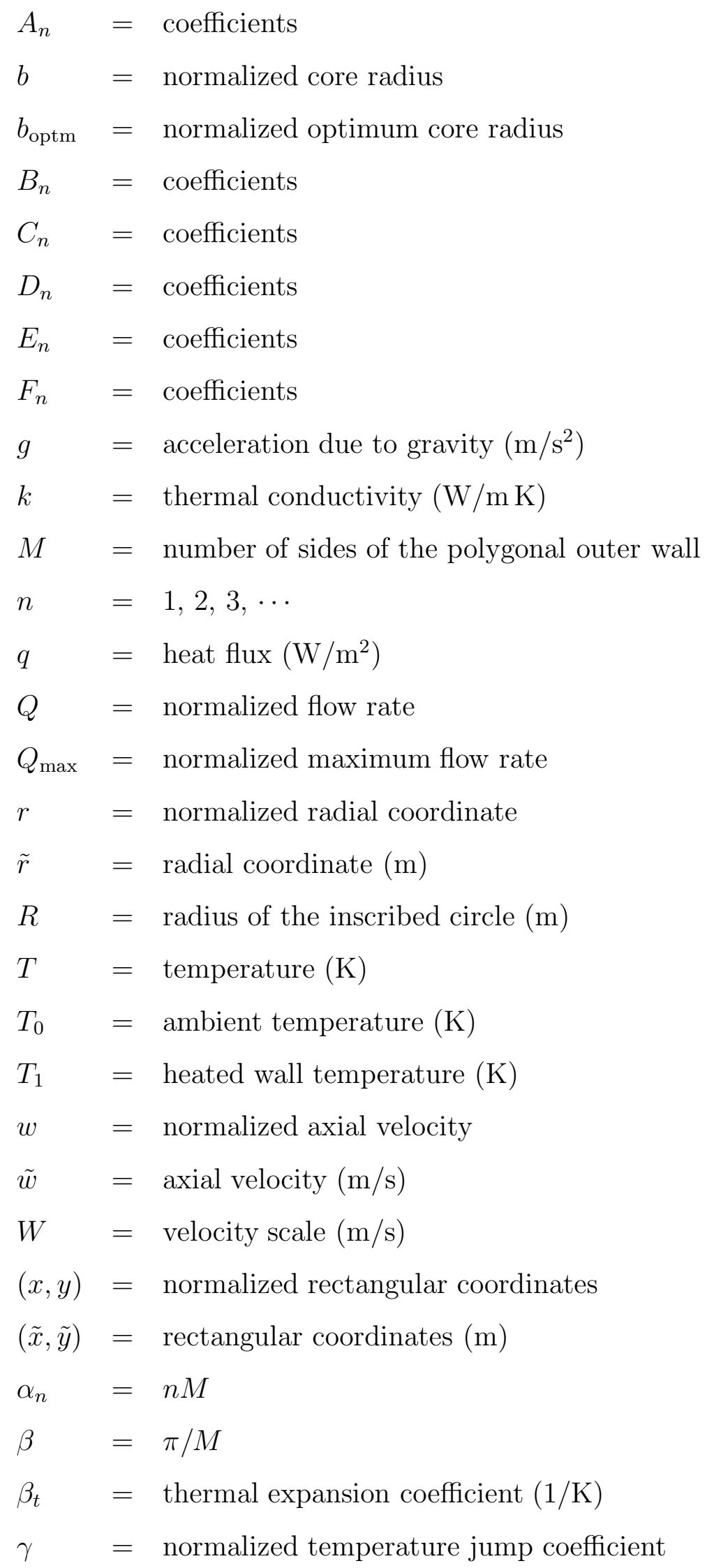




$$
\begin{aligned}
\tilde{\gamma} & =\text { temperature jump coefficient }(\mathrm{m}) \\
\theta & =\text { angular coordinate } \\
\lambda & =\text { normalized slip length } \\
\tilde{\lambda} & =\operatorname{slip} \text { length }(\mathrm{m}) \\
\nu & =\text { kinematic viscosity }\left(\mathrm{m}^{2} / \mathrm{s}\right) \\
\tau & =\text { normalized temperature }
\end{aligned}
$$

\section{Introduction}

It is often essential to consider flow in a micro-duct where the no-slip boundary condition is partially relaxed, or flow with partial slip on boundaries. Rarefied gas flow and flow of a liquid over a superhydrophobic surface in microchannels are typical examples of slip flow. When the flow involves heat transfer, there is a slip not only in velocity, but also in temperature, so-called temperature jump, on a fluid-solid boundary.

Convective heat transfer with velocity slip and temperature jump has been studied by several authors. Free convective gas microflow in a vertical asymmetrically heated slit channel was analytically studied by Chen and Weng [1] for walls at uniform wall temperature (UWT), and by Weng and Chen [2] for walls at uniform heat flux (UHF). Similar problems but for mixed convection were solved by Avci and Aydin [3] and Panda and Moulic [4]. Numerical studies were performed by Buonomo and Manca [5,6] on steady-state or transient natural convection in a vertical microchannel that is asymmetrically or symmetrically heated at UHF. A numerical study on slip-flow forced convection in a micro-duct of semicircular cross-section with temperature jump boundary condition was presented by Languri and Hooman [7].

Recently, Wang and Ng [8] looked into fully-developed free convection in a vertical parallelplate microchannel, which has one wall exhibiting superhydrophobic slip and temperature jump, while the other wall is a normal no-slip surface, and the heating is applied to only one of the two walls. They found that there exists a critical value of the temperature jump coefficient, which is a function of the slip length, above which the flow rate will be larger by heating the no-slip surface than by heating the superhydrophobic surface, whether the heated surface is at UWT or at UHF. Ng and Wang [9] further examined free convection in a 
vertical micro-annulus with superhydrophobic slip and temperature jump. They determined the optimum core radius for maximum flow rate, and investigated the singular increase of the flow rate for very small core radius, as functions of the velocity slip and temperature jump.

This paper aims to develop analytical solutions for fully-developed natural convection in a vertical polygonal duct with a heated circular core under the effect of velocity slip and temperature jump on the inner and outer walls. Heating by either UWT or UHF at the core wall is considered. Polygonal cross sections such as the square and hexagonal shapes can facilitate stacking, and thereby space utilization.

Convection (natural or forced) in polygonal ducts has been previously studied by Wang [1012], but velocity and thermal slip was not considered in these works. Slip flow with thermal jump arising from natural convection in a polygonal duct with a heated core has not been studied thus far. This has motivated the present study.

From what has been found by Wang $[12,13]$ and $\mathrm{Ng}$ and Wang [9], there always exists an optimum core size for maximum flow in natural convection in a duct with a heated core. This paper aims to determine the optimum core size as a function of the following: (i) slip length, (ii) temperature jump coefficient, (iii) number of sides of the polygon, and (iv) whether the heated core is at UWT or UHF. We shall look into how the temperature and velocity distributions are affected by the velocity slip and temperature jump. We shall also show that the temperature jump can have opposite effects on the flow rate, depending on whether the core wall is at UWT or UHF. Following Wang [10-12], we solve the present problem semi-analytically by the methods of eigenfunction expansion and boundary collocation.

\section{Case I: Heating by Uniform Wall Temperature (UWT)}

Consider steady fully-developed free convection in a long open vertical polygonal duct, composed of a heated circular core of radius $b R$ and an outer wall having the shape of an $M$-sided regular polygon, where $M \geq 3, R$ is the radius of the inscribed circle of the polygon (i.e., distance from the center of the duct to the mid-point of an edge of the polygon), and $0<b<1$ is a numerical factor representing the size of the inner core relative to $R$. Upon normalization 
by $R$, the non-dimensional core radius is $b$; see below. The outer wall is maintained at ambient temperature $T_{0}$, and the inner wall is uniformly heated to a higher temperature $T_{1}$. See Fig. 1 for a definition sketch of the problem. We assume that the inner and outer walls are surfaces with the same effective slip length $\tilde{\lambda}$ and temperature jump coefficient $\tilde{\gamma}$. For rarefied gas flow, the slip and temperature jump are known to depend on the Knudsen number and the thermal and tangential momentum accommodation coefficients, representing the effects of rarefaction and fluid-wall interaction [14]. For convection over a superhydrophobic surface, the slip and temperature jump depend mainly on the micro-texture of the surface [15].

Given long enough length of the channel, a thermally fully-developed region is established, where the temperature $T(\tilde{r}, \theta)$ is independent of vertical position. The flow is fully developed as well, with an upward velocity $\tilde{w}(\tilde{r}, \theta)$, where $(\tilde{r}, \theta)$ are the radial and azimuthal coordinates. By periodicity and symmetry, it will be sufficient to develop solutions for one half of a sector of the polygon enclosed by the radii at $\theta=0$ and $\theta=\beta$, where $\beta=\pi / M$; see Fig. $1(\mathrm{~b})$. The inner boundary is a circle of radius $\tilde{r}=b R$, while the outer boundary is a straight line segment given by $\tilde{x}=R, 0<\tilde{y}<R \tan \beta$, where $(\tilde{x}, \tilde{y})=\tilde{r}(\cos \theta, \sin \theta)$.

In the absence of forced convection, the dynamic pressure gradient is zero. Using the Boussinesq approximation, the energy and momentum equations are as follows:

$$
\begin{gathered}
\tilde{\nabla}^{2} T=0 \\
g \beta_{t}\left(T-T_{0}\right)+\nu \tilde{\nabla}^{2} \tilde{w}=0,
\end{gathered}
$$

where

$$
\tilde{\nabla}^{2}=\frac{1}{\tilde{r}} \frac{\partial}{\partial \tilde{r}}\left(\tilde{r} \frac{\partial}{\partial \tilde{r}}\right)+\frac{1}{\tilde{r}^{2}} \frac{\partial^{2}}{\partial \theta^{2}}
$$

and $g$ is the acceleration due to gravity, $\beta_{t}$ is the thermal expansion coefficient, and $\nu$ is the kinematic viscosity of the fluid.

Let us introduce the following dimensionless variables:

$$
w=\tilde{w} / W, \quad \tau=\left(T-T_{0}\right) /\left(T_{1}-T_{0}\right), \quad(r, x, y, \lambda, \gamma)=(\tilde{r}, \tilde{x}, \tilde{y}, \tilde{\lambda}, \tilde{\gamma}) / R,
$$

where $W=g \beta_{t}\left(T_{1}-T_{0}\right) R^{2} / \nu$ is the characteristic velocity for the flow, and $\lambda$ and $\gamma$ are respectively the normalized slip length and normalized temperature jump coefficient. In 
terms of these dimensionless variables, the energy and momentum equations become

$$
\begin{gathered}
\nabla^{2} \tau=0, \\
\nabla^{2} w+\tau=0,
\end{gathered}
$$

where $\nabla^{2}=R^{2} \tilde{\nabla}^{2}$

On the inner wall $(r=b)$, the temperature jump condition is

$$
\left(1-\tau+\gamma \frac{\partial \tau}{\partial r}\right)_{r=b}=0
$$

and the partial-slip condition for the flow is

$$
\left(w-\lambda \frac{\partial w}{\partial r}\right)_{r=b}=0
$$

On the outer wall $(x=1,0<y<\tan \beta)$, the temperature jump condition is

$$
\left(\tau+\gamma \frac{\partial \tau}{\partial x}\right)_{x=1}=0
$$

and the partial slip condition is

$$
\left(w+\lambda \frac{\partial w}{\partial r}\right)_{x=1}=0
$$

By symmetry, the temperature and velocity gradients are zero azimuthally along the two bounding radii:

$$
\begin{aligned}
& \left.\frac{\partial \tau}{\partial \theta}\right|_{\theta=0}=\left.\frac{\partial \tau}{\partial \theta}\right|_{\theta=\beta}=0 \\
& \left.\frac{\partial w}{\partial \theta}\right|_{\theta=0}=\left.\frac{\partial w}{\partial \theta}\right|_{\theta=\beta}=0 .
\end{aligned}
$$

\section{$2.1 \quad$ Temperature Field}

The solution to Eq. (5) that satisfies the boundary conditions at $r=b, \theta=0$ and $\theta=\beta$ is obtainable after some algebra as follows:

$$
\tau(r, \theta)=1+A_{0}\left[\ln \left(\frac{r}{b}\right)+\frac{\gamma}{b}\right]+\sum_{n=1}^{\infty} A_{n} \cos \left(\alpha_{n} \theta\right) \tau_{n}(r)
$$

where $\alpha_{n}=n M, A_{0,1,2, \ldots}$ are undetermined coefficients, and

$$
\tau_{n}(r)=\left(\frac{\gamma \alpha_{n}}{b}+1\right)\left(\frac{r}{b}\right)^{\alpha_{n}}+\left(\frac{\gamma \alpha_{n}}{b}-1\right)\left(\frac{b}{r}\right)^{\alpha_{n}} \quad \text { for } n=1,2, \cdots
$$


The remaining boundary condition to be satisfied is the temperature jump condition at the outer wall: Eq. (9), which in terms of the polar coordinates is written as

$$
\tau+\gamma\left(\cos \theta \frac{\partial \tau}{\partial r}-\frac{\sin \theta}{r} \frac{\partial \tau}{\partial \theta}\right)=0 \quad \text { on } r=\sec \theta, 0<\theta<\beta .
$$

The infinite series in Eq. (13) is truncated to $N-1$ terms. The $N$ unknown coefficients $A_{0}, A_{1}$, $\cdots, A_{N-1}$ are determined by the method of point collocation. The above boundary condition is satisfied at $N$ discrete points evenly distributed on the outer wall $(x=1,0<y<\tan \beta)$. This results in $N$ linear algebraic equations, which can be solved for the $N$ unknowns.

\subsection{Flow Field}

With Eq. (13), the momentum equation (6) becomes

$$
\frac{1}{r} \frac{\partial}{\partial r}\left(r \frac{\partial w}{\partial r}\right)+\frac{1}{r^{2}} \frac{\partial^{2} w}{\partial \theta^{2}}=-1-A_{0}\left[\ln \left(\frac{r}{b}\right)+\frac{\gamma}{b}\right]-\sum_{n=1}^{\infty} A_{n} \cos \left(\alpha_{n} \theta\right) \tau_{n}(r) .
$$

Let us expand the velocity as follows:

$$
w(r, \theta)=w_{0}(r)+\sum_{n=1}^{\infty} \cos \left(\alpha_{n} \theta\right) w_{n}(r),
$$

such that

$$
\frac{1}{r} \frac{\mathrm{d}}{\mathrm{d} r}\left(r \frac{\mathrm{d} w_{0}}{\mathrm{~d} r}\right)=-1-A_{0}\left[\ln \left(\frac{r}{b}\right)+\frac{\gamma}{b}\right]
$$

and

$$
\frac{1}{r} \frac{\mathrm{d}}{\mathrm{d} r}\left(r \frac{\mathrm{d} w_{n}}{\mathrm{~d} r}\right)-\frac{\alpha_{n}^{2}}{r^{2}} w_{n}=-A_{n} \tau_{n}(r)
$$

where $\tau_{n}$ is given in Eq. (14).

Upon integrating Eq. (18) twice with respect to $r$ and using the slip condition (8) at $r=b$, we get the following solution for $w_{0}$ :

$$
\begin{aligned}
w_{0}(r)= & \left(A_{0}-1-A_{0} \frac{\gamma}{b}\right) \frac{\left(r^{2}-b^{2}\right)}{4}-A_{0} \frac{r^{2}}{4} \ln \left(\frac{r}{b}\right)+B_{0} \ln \left(\frac{r}{b}\right) \\
& -\lambda\left[\frac{b}{2}+A_{0}\left(\frac{\gamma}{2}-\frac{b}{4}\right)-\frac{B_{0}}{b}\right],
\end{aligned}
$$

where $B_{0}$ is an unknown constant to be determined. 
With $\tau_{n}$ given by Eq. (14), the general solution to Eq. (19) is readily found to be

$$
\begin{aligned}
w_{n}(r)= & C_{n}\left(\frac{r}{b}\right)^{\alpha_{n}}+B_{n}\left(\frac{b}{r}\right)^{\alpha_{n}}+A_{n} \frac{b\left(\gamma \alpha_{n}+b\right)}{\alpha_{n}^{2}-\left(\alpha_{n}+2\right)^{2}}\left(\frac{r}{b}\right)^{\alpha_{n}+2} \\
& +A_{n} \frac{b\left(\gamma \alpha_{n}-b\right)}{\alpha_{n}^{2}-\left(\alpha_{n}-2\right)^{2}}\left(\frac{b}{r}\right)^{\alpha_{n}-2},
\end{aligned}
$$

where the first two terms are the complementary solution, and the last two terms are the particular solution to Eq. (19). Applying the inner-wall slip condition (8), we may obtain

$$
\begin{aligned}
C_{n}= & -B_{n}\left(\frac{1+\lambda \alpha_{n} / b}{1-\lambda \alpha_{n} / b}\right)-A_{n} \frac{\left[b-\lambda\left(\alpha_{n}+2\right)\right]\left(\gamma \alpha_{n}+b\right)}{\left[\alpha_{n}^{2}-\left(\alpha_{n}+2\right)^{2}\right]\left(1-\lambda \alpha_{n} / b\right)} \\
& -A_{n} \frac{\left[b+\lambda\left(\alpha_{n}-2\right)\right]\left(\gamma \alpha_{n}-b\right)}{\left[\alpha_{n}^{2}-\left(\alpha_{n}-2\right)^{2}\right]\left(1-\lambda \alpha_{n} / b\right)}
\end{aligned}
$$

where $B_{n}(n=1,2, \cdots)$ are unknown coefficients yet to be determined. Putting $C_{n}$ back into Eq. (21), we may write

$$
\begin{aligned}
w_{n}(r)= & B_{n}\left\{-\left(\frac{1+\lambda \alpha_{n} / b}{1-\lambda \alpha_{n} / b}\right)\left(\frac{r}{b}\right)^{\alpha_{n}}+\left(\frac{b}{r}\right)^{\alpha_{n}}\right\}+A_{n}\left\{\frac{b\left(\gamma \alpha_{n}+b\right)}{\alpha_{n}^{2}-\left(\alpha_{n}+2\right)^{2}}\left(\frac{r}{b}\right)^{\alpha_{n}+2}\right. \\
& +\frac{b\left(\gamma \alpha_{n}-b\right)}{\alpha_{n}^{2}-\left(\alpha_{n}-2\right)^{2}}\left(\frac{b}{r}\right)^{\alpha_{n}-2}-\left(\frac{\left[b-\lambda\left(\alpha_{n}+2\right)\right]\left(\gamma \alpha_{n}+b\right)}{\left[\alpha_{n}^{2}-\left(\alpha_{n}+2\right)^{2}\right]\left(1-\lambda \alpha_{n} / b\right)}\right. \\
& \left.\left.+\frac{\left[b+\lambda\left(\alpha_{n}-2\right)\right]\left(\gamma \alpha_{n}-b\right)}{\left[\alpha_{n}^{2}-\left(\alpha_{n}-2\right)^{2}\right]\left(1-\lambda \alpha_{n} / b\right)}\right)\left(\frac{r}{b}\right)^{\alpha_{n}}\right\} \quad \text { for } n=1,2, \cdots
\end{aligned}
$$

The series in Eq. (17) being truncated to $N-1$ terms, the $N$ unknown coefficients $B_{0}$, $B_{1}, \cdots, B_{N-1}$ can be determined using the method of point collocation again. The partialslip condition (10) is to be satisfied at $N$ equally spaced points on the outer wall $(x=1$, $0<y<\tan \beta)$. This generates $N$ linear algebraic equations, which can be solved for the $N$ unknowns.

The volume flow rate through the duct is given by

$$
Q=2 M \int_{0}^{\beta} \int_{b}^{\sec \theta} w r \mathrm{~d} r \mathrm{~d} \theta
$$

which can be evaluated numerically by Simpson's formula.

\section{Case II: Heating by Uniform Heat Flux (UHF)}

Consider the same problem as formulated in case I, except that the heating is now due to a constant heat flux $q$ applied to the inner wall, while the outer wall is maintained at ambient 
temperature $T_{0}$. The dimensionless momentum and energy equations are still given by Eqs. (6) and (5), but the normalization is now changed to

$$
w=\tilde{w} / W, \quad \tau=\left(T-T_{0}\right) /(q R / k),
$$

where $W=g \beta_{t} q R^{3} / \nu k$ is the characteristic velocity for the flow, and $k$ is the thermal conductivity of the fluid.

On the inner wall $(r=b)$, the thermal boundary condition is now changed to

$$
\left.\frac{\partial \tau}{\partial r}\right|_{r=b}=-1
$$

which is unaffected by the temperature jump. The other boundary conditions remain the same as those in case (I), as given in Eqs. (8)-(12).

\subsection{Temperature Field}

In this case, the solution to Eq. (5) that satisfies the boundary conditions at $r=b, \theta=0$ and $\theta=\beta$ is obtainable as follows:

$$
\tau(r, \theta)=D_{0}-b \ln \left(\frac{r}{b}\right)+\sum_{n=1}^{\infty} D_{n} \cos \left(\alpha_{n} \theta\right) \tau_{n}(r)
$$

where $\alpha_{n}=n M, D_{0,1,2, \ldots}$ are undetermined coefficients, and

$$
\tau_{n}(r)=\left(\frac{r}{b}\right)^{\alpha_{n}}+\left(\frac{b}{r}\right)^{\alpha_{n}} \quad \text { for } n=1,2, \cdots .
$$

Again, by truncating the series in Eq. (27) to $N-1$ terms, the $N$ coefficients $D_{0}, D_{1}, \cdots, D_{N-1}$ can be determined by the method of point collocation, as explained above. The boundary condition at the outer wall is satisfied at $N$ discrete points evenly distributed on the line: $x=1,0<y<\tan \beta$.

\subsection{Flow Field}

With Eq. (27), the momentum equation (6) becomes

$$
\frac{1}{r} \frac{\partial}{\partial r}\left(r \frac{\partial w}{\partial r}\right)+\frac{1}{r^{2}} \frac{\partial^{2} w}{\partial \theta^{2}}=-D_{0}+b \ln \left(\frac{r}{b}\right)-\sum_{n=1}^{\infty} D_{n} \cos \left(\alpha_{n} \theta\right) \tau_{n}(r)
$$


Let us expand the velocity as follows:

$$
w(r, \theta)=w_{0}(r)+\sum_{n=1}^{\infty} \cos \left(\alpha_{n} \theta\right) w_{n}(r),
$$

such that

$$
\frac{1}{r} \frac{\mathrm{d}}{\mathrm{d} r}\left(r \frac{\mathrm{d} w_{0}}{\mathrm{~d} r}\right)=-D_{0}+b \ln \left(\frac{r}{b}\right)
$$

and

$$
\frac{1}{r} \frac{\mathrm{d}}{\mathrm{d} r}\left(r \frac{\mathrm{d} w_{n}}{\mathrm{~d} r}\right)-\frac{\alpha_{n}^{2}}{r^{2}} w_{n}=-D_{n} \tau_{n}(r)
$$

where $\tau_{n}$ is given in Eq. (28).

Upon integrating Eq. (31) twice with respect to $r$ and using the slip condition (8) at $r=b$, we get the following solution for $w_{0}$ :

$$
\begin{aligned}
w_{0}(r)= & -\left(D_{0}+b\right) \frac{\left(r^{2}-b^{2}\right)}{4}+b \frac{r^{2}}{4} \ln \left(\frac{r}{b}\right)+E_{0} \ln \left(\frac{r}{b}\right) \\
& -\lambda\left[D_{0} \frac{b}{2}+\frac{b^{2}}{4}-\frac{E_{0}}{b}\right],
\end{aligned}
$$

where $E_{0}$ is an unknown constant to be determined.

With $\tau_{n}$ given by Eq. (28), the general solution to Eq. (32) is readily found to be

$$
\begin{aligned}
w_{n}(r)= & F_{n}\left(\frac{r}{b}\right)^{\alpha_{n}}+E_{n}\left(\frac{b}{r}\right)^{\alpha_{n}}+D_{n} \frac{b^{2}}{\alpha_{n}^{2}-\left(\alpha_{n}+2\right)^{2}}\left(\frac{r}{b}\right)^{\alpha_{n}+2} \\
& +D_{n} \frac{b^{2}}{\alpha_{n}^{2}-\left(\alpha_{n}-2\right)^{2}}\left(\frac{b}{r}\right)^{\alpha_{n}-2},
\end{aligned}
$$

where the first two terms are the complementary solution, and the last two terms are the particular solution to Eq. (32). Applying the inner-wall slip condition (8), we may obtain

$$
\begin{aligned}
F_{n}= & -E_{n}\left(\frac{1+\lambda \alpha_{n} / b}{1-\lambda \alpha_{n} / b}\right)-D_{n} \frac{b^{2}-\lambda b\left(\alpha_{n}+2\right)}{\left[\alpha_{n}^{2}-\left(\alpha_{n}+2\right)^{2}\right]\left(1-\lambda \alpha_{n} / b\right)} \\
& -D_{n} \frac{b^{2}+\lambda b\left(\alpha_{n}-2\right)}{\left[\alpha_{n}^{2}-\left(\alpha_{n}-2\right)^{2}\right]\left(1-\lambda \alpha_{n} / b\right)},
\end{aligned}
$$

where $E_{n}(n=1,2, \cdots)$ are unknown coefficients yet to be determined. Putting $F_{n}$ back into Eq. (34), we may write

$$
\begin{aligned}
w_{n}(r)= & E_{n}\left\{-\left(\frac{1+\lambda \alpha_{n} / b}{1-\lambda \alpha_{n} / b}\right)\left(\frac{r}{b}\right)^{\alpha_{n}}+\left(\frac{b}{r}\right)^{\alpha_{n}}\right\}+D_{n}\left\{\frac{b^{2}}{\alpha_{n}^{2}-\left(\alpha_{n}+2\right)^{2}}\left(\frac{r}{b}\right)^{\alpha_{n}+2}\right. \\
& +\frac{b^{2}}{\alpha_{n}^{2}-\left(\alpha_{n}-2\right)^{2}}\left(\frac{b}{r}\right)^{\alpha_{n}-2}-\left(\frac{b^{2}-\lambda b\left(\alpha_{n}+2\right)}{\left[\alpha_{n}^{2}-\left(\alpha_{n}+2\right)^{2}\right]\left(1-\lambda \alpha_{n} / b\right)}\right. \\
& \left.\left.+\frac{b^{2}+\lambda b\left(\alpha_{n}-2\right)}{\left[\alpha_{n}^{2}-\left(\alpha_{n}-2\right)^{2}\right]\left(1-\lambda \alpha_{n} / b\right)}\right)\left(\frac{r}{b}\right)^{\alpha_{n}}\right\} \quad \text { for } n=1,2, \cdots
\end{aligned}
$$


As in case (I), with the series in Eq. (30) truncated to $N-1$ terms, the $N$ unknown coefficients $E_{0}, E_{1}, \cdots, E_{N-1}$ can be determined numerically using the method of point collocation by imposing the partial-slip condition (10) at $N$ equally spaced points on the outer wall $(x=1$, $0<y<\tan \beta)$.

The flow rate, given by the same integral as in Eq. (24), can then be found by evaluating the integral numerically.

\section{Circular Annular Duct}

In the limit of a large number of edges, $M \rightarrow \infty$, the polygonal duct will virtually become a circular duct. In this limiting case, the problem becomes independent of $\theta$, by which the energy and momentum Eqs. (5) and (6) can be solved analytically. They are

$$
\begin{gathered}
\tau(r)=C_{1} \ln (r)+C_{2}, \\
w(r)=-\frac{C_{1}}{4} r^{2} \ln (r)+\left(C_{1}-C_{2}\right) \frac{r^{2}}{4}+C_{3} \ln (r)+C_{4},
\end{gathered}
$$

where $C_{1}, \cdots, C_{4}$ are undetermined constants. The volume flow rate is then given by

$$
\begin{aligned}
Q= & 2 \pi \int_{b}^{1} w r \mathrm{~d} r \\
= & \pi\left\{\frac{C_{1}}{32}\left[1+b^{4}(4 \ln (b)-1)\right]+\frac{C_{1}-C_{2}}{8}\left(1-b^{4}\right)\right. \\
& \left.-\frac{C_{3}}{2}\left[1+b^{2}(2 \ln (b)-1)\right]+C_{4}\left(1-b^{2}\right)\right\}
\end{aligned}
$$

For case I (heating due to a uniform wall temperature at the inner wall), the boundary conditions (7)-(10) give

$$
\begin{gathered}
C_{1}=\frac{b}{b \ln (b)-\gamma(1+b)}, \\
C_{2}=-\gamma C_{1}, \\
C_{3}=\frac{b C_{1}}{4[b \ln (b)-\lambda(1+b)]}\left[(1+\gamma)\left(1-b^{2}\right)+\lambda(1+2 \gamma)(1+b)+b(b-2 \lambda) \ln (b)\right], \\
C_{4}=-\lambda C_{3}-\frac{C_{1}}{4}[1+\gamma+\lambda(1+2 \gamma)] .
\end{gathered}
$$

For case II (heating due to a uniform heat flux at the inner wall), the boundary condition (26) gives $C_{1}=-b$. The other boundary conditions are the same as those in case (I), and therefore $C_{2}, C_{3}$ and $C_{4}$ are related to $C_{1}$ by the same relationships as given above. 


\section{Discussion}

Let us first look into how the temperature jump and velocity slip will affect the temperature and flow fields. For $M=4$ (square duct), we compare the temperature distributions when $\gamma=0$ (i.e., no temperature jump), as shown in Fig. 2, with those when $\gamma=1$ (i.e., a finite temperature jump), as shown in Fig. 3. We choose to show the results for the specific values of $b=0.5$ and $b=0.9$, as these results are illustrative of the limiting cases where the inner core is sufficiently far from, or close to, the outer wall, respectively. The following observations can be inferred from the figures. First, when the core wall is at UWT, the temperature jump is to lower the temperature near the heated core, but is to raise the temperature near the outer wall. This is because the jump will impede the input of heat from the hotter wall into the fluid, but will on the other hand prevent the loss of heat from the fluid to the cooler wall. As a result, the temperature is more uniformly distributed in the fluid under the presence of a temperature jump on the walls. Second, if the core wall is at UHF, the temperature jump is to increase the temperature everywhere in the fluid. This is owing to the fact the constant flux condition is independent of the temperature jump. Hence, in this case, the temperature jump serves only to prevent heat loss at the outer wall. This also suggests that the temperature jump can have a more positive effect on the free convection when the core is heated by uniform flux than by uniform wall temperature. Third, in the presence of temperature jump, the temperature is more axisymmetrically distributed than that without temperature jump. This is especially true for a small core, say $b=0.5$, for which the temperature contours remain nearly concentric circles throughout the fluid domain, despite the shape of the outer wall. Fourth, for a larger core, say $b=0.9$, the temperature becomes more nonuniformly distributed on the inner wall, when the core wall is at UHF. It is interesting to note that the maximum temperature on the inner wall occurs at $\theta=\pi / 4$ when there is no temperature jump (Fig. 2(d)), but at $\theta=0$ or $\pi / 2$ when there is a finite jump (Fig. $3(\mathrm{~d})$ ).

We then show the corresponding convection velocity distributions in Figs. 4 and 5, for $\gamma=\lambda=0$ (zero jump and slip) and $\gamma=\lambda=1$ (finite jump and slip), respectively. As expected, the slip at the walls is to increase the velocity across the entire channel. On comparing these two figures, one can see that the maximum velocity, which occurs somewhere in the middle of the widest part of the channel, is substantially increased owing to a finite 
velocity slip and temperature jump. The percentage increase depends on the type of heating and the core radius. For $b=0.5$, the maximum velocity is approximately 5 times that without slip and jump when the core is heated under UWT, but is 24 times when the heating is by UHF. For $b=0.9$, these two factors increase dramatically to 9 times and 70 times, respectively. These values suggest that the temperature jump and velocity slip will have a greater impact on the convection when the heating is by constant heat flux, and for a larger core radius. In addition, the flow is virtually stagnant near the corner of the outer wall (and also near the narrowest part of the channel for $b=0.9$ ) when the walls are non-slip. In the presence of slip, such stagnant zones no longer exist; fluid can flow through readily even the narrowest passage.

Qualitatively similar temperature and velocity distributions have been found for polygons of different numbers of sides $(M=3,6,8)$, but in the interest of space, the results are not shown here.

To further illustrate the effect of the temperature jump and the velocity slip on the flow, we show in Fig. 6 the flow rate $Q$ as a function of $\gamma$ and $\lambda$, where $M=4$ and $b=0.5$. Under the condition of UWT, the flow rate increases with a larger slip length, but decreases with a larger temperature jump coefficient. As explained above, the jump will in this case hinder the transfer of heat from the heated core to the fluid, and will result in an overall negative effect on the flow. From Fig. 6(a), one can infer that this negative effect is rather mild for a small slip length, but will be enhanced by a larger slip length. In contrast, when it is under the condition of UHF, the flow rate increases with either a larger slip length or a larger jump coefficient. Hence, under the combined effect of velocity slip and temperature jump, the flow rate can increase more appreciably when the heating is due to UHF than when it is due to UWT.

The flow rates for six types of polygons: $M=3,4,5,6,8, \infty$, as functions of the core radius $b$ are shown in Fig. 7, where $\lambda=\gamma=1$. In the lower limit $b=0$, the flow rates are zero because of the vanishing strength of the heat source. In the upper limit $b=1$, the flow rates also diminish to a small value because of the dwindling sectional area between the polygon and the core. There exists for each case an intermediate value of $b$, which is referred to as the optimum core radius $b_{\text {optm }}$, at which the flow rate is the maximum. One can see 
from Fig. 7 that as $M$ increases, both the maximum flow rate $Q_{\max }$ and the corresponding optimum core radius $b_{\text {optm }}$ will decrease. For future reference, we provide in Table 1 the values of the maximum flow rate $Q_{\max }$ and the corresponding optimum core radius $b_{\mathrm{optm}}$ for the cases shown in Fig. 7. These optimum values, which are determined either with the aid of Mathematica (for $M=\infty$ only) or by searching the maximum numerically, can serve as benchmarks for approximate or numerical methods solving similar problems in the future.

The maximum flow rate and the corresponding optimum core radius will change as the slip coefficients change. Figure 8 shows $Q_{\max }$ and $b_{\text {optm }}$ as functions of $\gamma$ and $\lambda$, where $\gamma=\lambda$, and $M=3,4,6, \infty$. The values of $Q_{\max }$ and $b_{\text {optm }}$ for the no-slip limit $(\gamma=\lambda=0)$ agree exactly with those obtained previously by Wang [12]. In all cases shown in this figure, both $Q_{\max }$ and $b_{\text {optm }}$ increase monotonically with increasing $\gamma$ and $\lambda$, for either UWT or UHF. Under the condition of UWT, $Q_{\max }$ increases nearly linearly with the slip and jump coefficients. Under the condition of UHF, $Q_{\max }$ is subject to a more dramatic nonlinear increase with these coefficients. Consistent with our observation above, the flow is more sensitively affected by the velocity slip and temperature jump when the heating is due to UHF than when it is due to UWT. Also, for the same channel properties, the optimum core radius is always higher when the core wall is at UHF than when it is at UWT.

\section{Conclusions}

Fully-developed natural convection under the combined effect of velocity slip and temperature jump in an open vertical polygonal duct with a heated circular core has been solved by the methods of eigenfunction expansion and point match. The core is heated with its wall either at uniform wall temperature (UWT) or uniform heat flux (UHF). The present problem applies to both superhydrophobic slip or rarefied gas slip flows. We have shown that the temperature jump will have a positive effect on the flow when the core wall is at UHF. In contrast, when the core wall is at UWT, the flow rate is decreased by the temperature jump; such a negative effect is enhanced by a velocity slip. We have also determined in particular the optimum core radius for maximum flow rate as a function of the slip coefficients and the number of sides of the polygon. Our results presented in Table 1 and Fig. 8 are the key findings of this study, 
and should serve as a benchmark for future studies.

The present problem is for fully-developed flow, which applies to an infinitely long duct. It is desirable if the present problem is extended to developing free convection slip flow in the entrance region of a duct of finite length.

\section{Acknowledgments}

Financial support was given by the Research Grants Council of the Hong Kong Special Administrative Region, China, through Project No. HKU 715609E.

\section{References}

[1] Chen, C. K., and Weng, H. C., "Natural Convection in a Vertical Microchannel," Journal of Heat Transfer, Vol. 127, No. 9, 2005, pp. 1053-1056.

[2] Weng, H. C., and Chen, C. K., "On the Importance of Thermal Creep in Natural Convective Gas Microflow With Wall Heat Fluxes," Journal of Physics D: Applied Physics, Vol. 41, No. 11, 2008, Paper 115501.

[3] Avci, M., and Aydin, O., "Mixed Convection in a Vertical Parallel Plate Microchannel," Journal of Heat Transfer, Vol. 129, No. 2, 2007, pp. 162-166.

[4] Panda, H. S., and Moulic, S. G., "An Analytical Solution for Natural Convective Gas Microflow in a Tall Vertical Enclosure," Proceedings of IMechE, Part C: Journal of Mechanical Engineering Science, Vol. 225, No. 1, 2011, pp. 145-154.

[5] Buonomo, B., and Manca, O., "Natural Convection Slip Flow in a Vertical Microchannel Heated at Uniform Heat Flux," International Journal of Thermal Sciences, Vol. 49, No. 8, 2010, pp. 1333-1344.

[6] Buonomo, B., and Manca, O., "Transient Natural Convection in a Vertical Microchannel Heated at Uniform Heat Flux," International Journal of Thermal Sciences, Vol. 56, No. 1, 2012, pp. 35-47. 
[7] Languri, E. M., and Hooman, K., "Slip Flow Forced Convection in a Microchannel With Semi-Circular Cross-Section," International Communications in Heat and Mass Transfer, Vol. 38, No. 2, 2011, pp. 139-143.

[8] Wang, C. Y., and Ng, C. O., "Natural Convection in a Vertical Slit Microchannel With Superhydrophobic Slip and Temperature Jump," Journal of Heat Transfer, Vol. 136, No. 3, 2014, Paper 034502.

[9] Ng, C. O., and Wang, C. Y., "Natural Convection in a Vertical Microannulus With Superhydrophobic Slip and Temperature Jump," Journal of Thermophysics and Heat Transfer, Vol. 28, No. 2, 2014, pp. 287-294.

[10] Wang, C. Y., "Forced Convection in a Polygonal Duct With a Circular Core," Journal of Heat Transfer, Vol. 133, No. 4, 2011, Paper 044506.

[11] Wang, C. Y., "Flow and Heat Transfer Through a Polygonal Duct Filled With a Porous Medium," Transport in Porous Media, Vol. 90, No. 2, 2011, pp. 321-332.

[12] Wang, C. Y., "Optimum Natural Convection in a Porous Medium Between a Vertical Polygonal Duct and a Heated Core," Journal of Heat Transfer, Vol. 134, No. 8, 2012, Paper 084501.

[13] Wang, C. Y., "Optimization of Natural Convection in Open Vertical Ducts with Heated Cores," Journal of Thermophysics and Heat Transfer, Vol. 24, No. 3, 2010, pp. 669-672.

[14] Karniadakis, G., Beskok, A., and Aluru, N., Microflows and Nanoflows Fundamentals and Simulation, Springer, New York, 2005.

[15] Ng, C. O., and Wang, C. Y., "Temperature Jump Coefficient for Superhydrophobic Surfaces," Journal of Heat Transfer, Vol. 136, No. 6, 2014, Paper 064501.

\section{Captions}

Table 1 The maximum flow rate $Q_{\max }$ and the corresponding optimum core radius $b_{\text {optm }}$ for the cases shown in Fig. 7. 
Figure 1 (a) Fully-developed natural convection in an open-ended, vertical, regular polygonal duct with a circular core, the wall of which is either at uniform wall temperature (UWT) or uniform heat flux (UHF), and the polygonal wall is maintained at ambient temperature. (b) The domain of analysis, which is bounded by the radii at $\theta=0$ and $\theta=\beta=\pi / M$, the inner wall at $r=b$, and the outer wall at $x=1$, where $M$ is the number of sides of the polygon, and the lengths are normalized by the radius of the inscribed circle of the polygon.

Figure 2 Temperature distribution $\tau(x, y)$ for $M=4$ (square), $\gamma=0$ (i.e., no temperature jump), and (a) $b=0.5$, UWT; (b) $b=0.5$, UHF; (c) $b=0.9$, UWT; (d) $b=0.9$, UHF.

Figure 3 Temperature distribution $\tau(x, y)$ for $M=4$ (square), $\gamma=1$ (i.e., finite temperature jump), and (a) $b=0.5$, UWT; (b) $b=0.5$, UHF; (c) $b=0.9$, UWT; (d) $b=0.9$, UHF.

Figure 4 Velocity distribution $w(x, y)$ for $M=4$ (square), $\gamma=\lambda=0$ (i.e., no temperature jump and velocity slip), and (a) $b=0.5$, UWT; (b) $b=0.5$, UHF; (c) $b=0.9$, UWT; (d) $b=0.9, \mathrm{UHF}$.

Figure 5 Velocity distribution $w(x, y)$ for $M=4$ (square), $\gamma=\lambda=1$ (i.e., finite temperature jump and velocity slip), and (a) $b=0.5$, UWT; (b) $b=0.5$, UHF; (c) $b=0.9$, $\mathrm{UWT} ;$ (d) $b=0.9, \mathrm{UHF}$.

Figure 6 Flow rate $Q$ as a function of the temperature jump coefficient $\gamma$ and the slip length $\lambda$, for $M=4$ (square), $b=0.5$, and the core wall is at (a) UWT; (b) UHF.

Figure 7 Flow rate $Q$ as a function of the core radius $b$, for $M=3,4,5,6,8, \infty, \gamma=\lambda=1$, and the core wall is at (a) UWT; (b) UHF. Values of the curve peaks, which are marked by the open circles, are given in Table 1.

Figure 8 The maximum flow rate $Q_{\max }$ and the corresponding optimum core radius $b_{\text {optm }}$ as functions of the temperate jump coefficient $\gamma$ and the slip length $\lambda$, where $\gamma=\lambda$, for $M=3,4,6, \infty$, and the core wall at (a) UWT; (b) UHF. 
Table 1: The maximum flow rate $Q_{\max }$ and the corresponding optimum core radius $b_{\text {optm }}$ for the cases shown in Fig. 7.

\begin{tabular}{|c|c|c|}
\hline$M$ & $\mathrm{UWT}$ & $\mathrm{UHF}$ \\
\hline 3 & $0.5597(0.385)$ & $0.8246(0.416)$ \\
4 & $0.3900(0.333)$ & $0.6167(0.378)$ \\
5 & $0.3428(0.315)$ & $0.5554(0.363)$ \\
6 & $0.3220(0.307)$ & $0.5281(0.356)$ \\
8 & $0.3040(0.300)$ & $0.5041(0.349)$ \\
$\infty$ & $0.2841(0.291)$ & $0.4773(0.341)$ \\
\hline
\end{tabular}


(a)

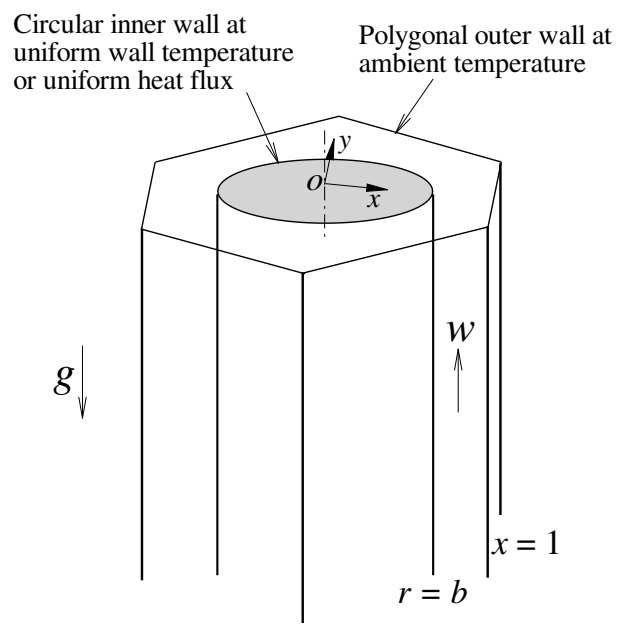

(b)

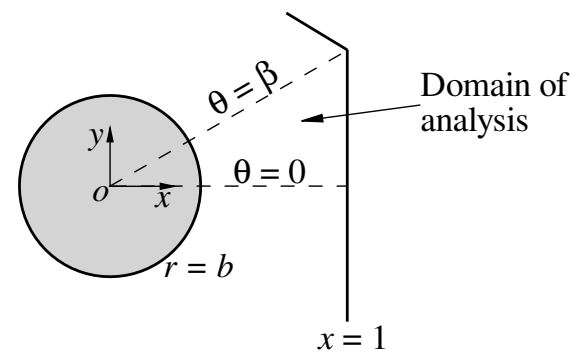

Figure 1: (a) Fully-developed natural convection in an open-ended, vertical, regular polygonal duct with a circular core, the wall of which is either at uniform wall temperature (UWT) or uniform heat flux (UHF), and the polygonal wall is maintained at ambient temperature. (b) The domain of analysis, which is bounded by the radii at $\theta=0$ and $\theta=\beta=\pi / M$, the inner wall at $r=b$, and the outer wall at $x=1$, where $M$ is the number of sides of the polygon, and the lengths are normalized by the radius of the inscribed circle of the polygon. 
(a) $b=0.5$, UWT

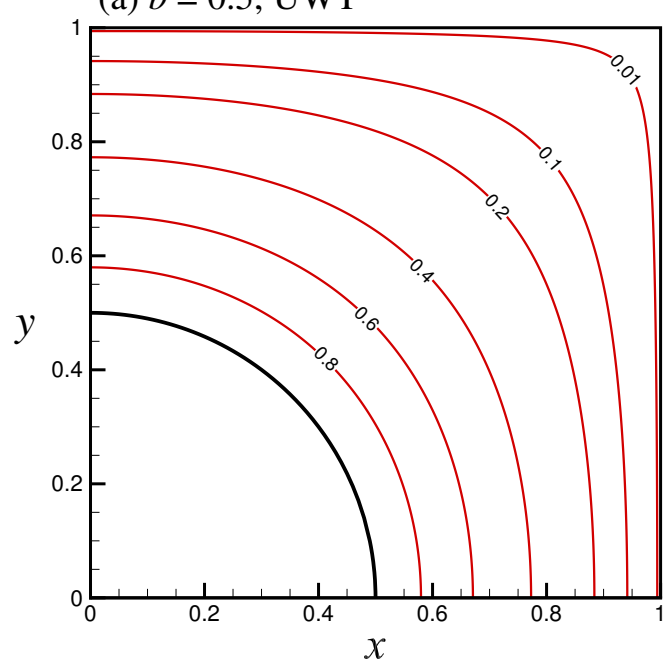

(c) $b=0.9$, UWT

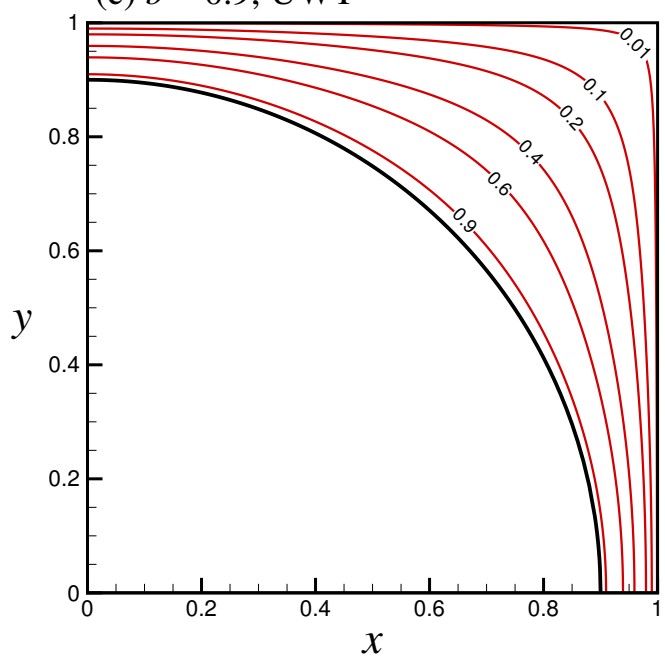

(b) $b=0.5$, UHF

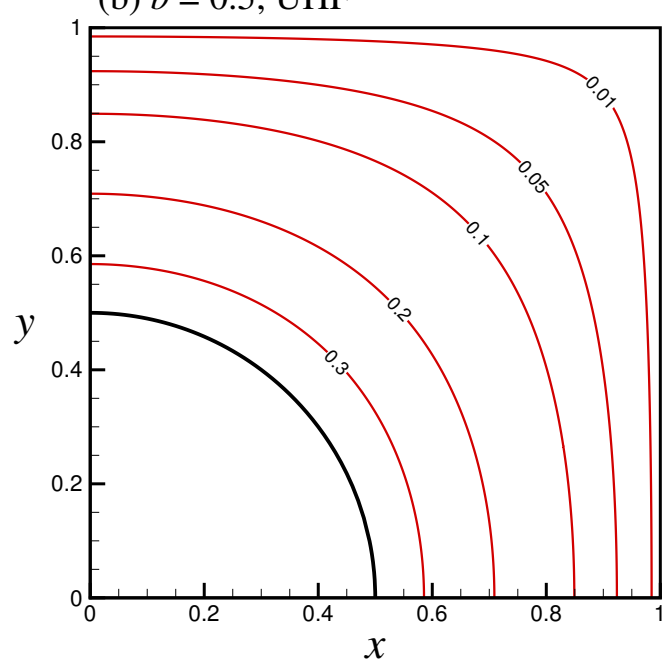

(d) $b=0.9$, UHF

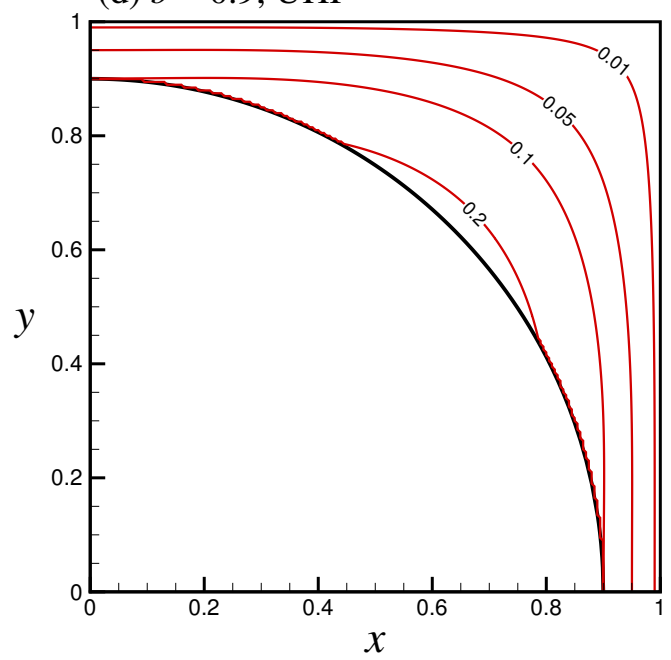

Figure 2: Temperature distribution $\tau(x, y)$ for $M=4$ (square), $\gamma=0$ (i.e., no temperature jump), and (a) $b=0.5$, UWT; (b) $b=0.5$, UHF; (c) $b=0.9$, UWT; (d) $b=0.9$, UHF. 
(a) $b=0.5$, UWT

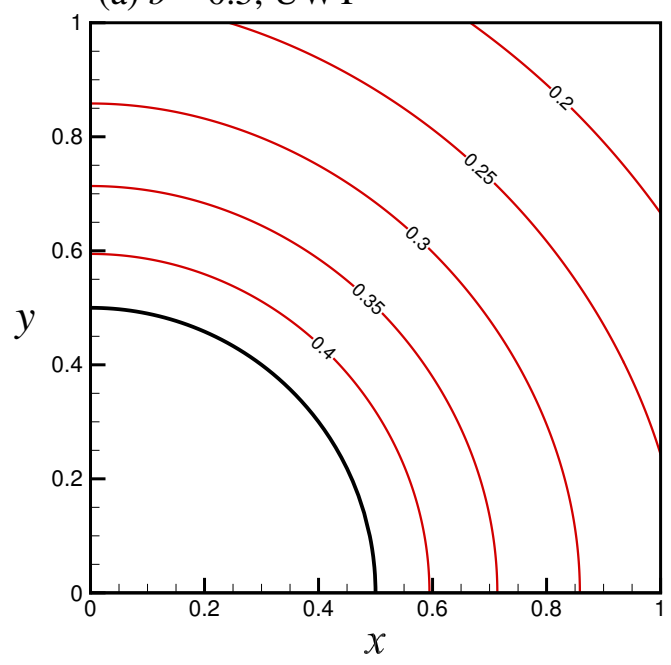

(c) $b=0.9$, UWT

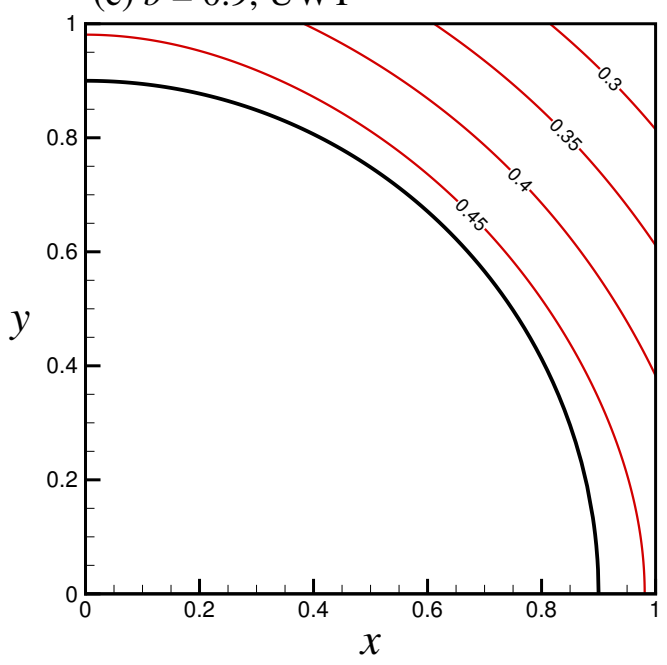

(b) $b=0.5$, UHF

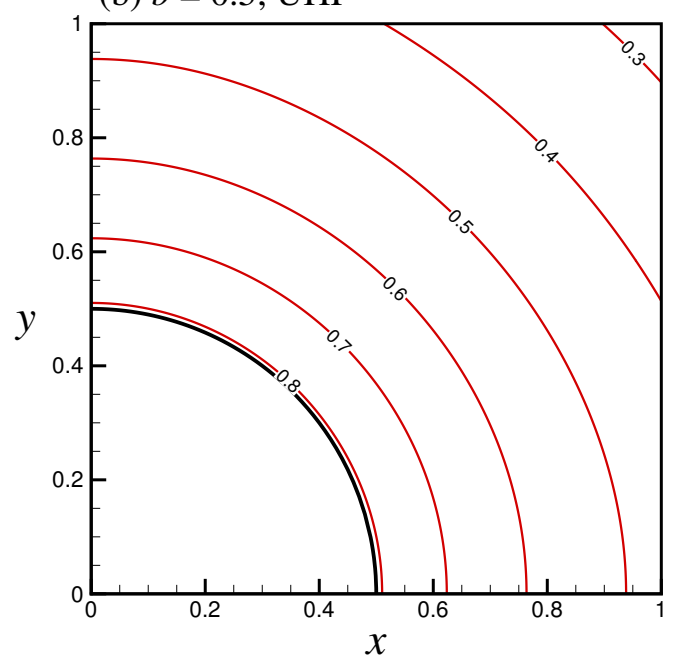

(d) $b=0.9$, UHF

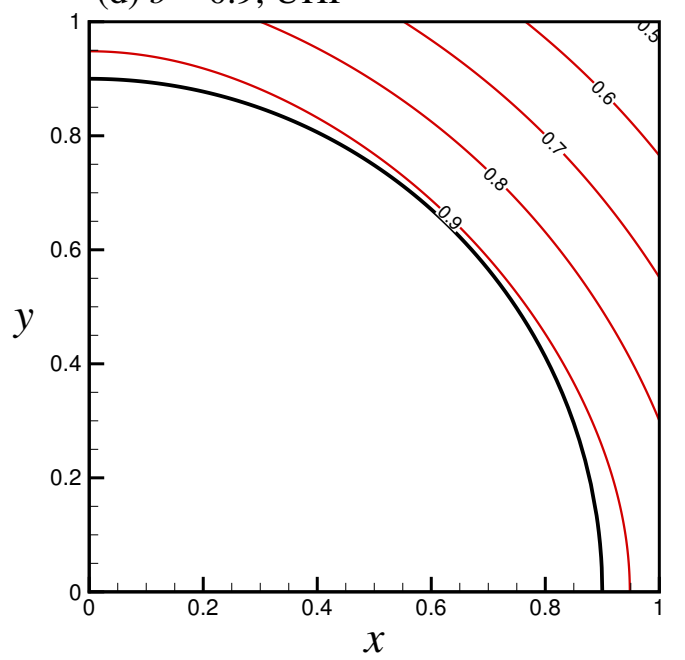

Figure 3: Temperature distribution $\tau(x, y)$ for $M=4$ (square), $\gamma=1$ (i.e., finite temperature jump), and (a) $b=0.5$, UWT; (b) $b=0.5$, UHF; (c) $b=0.9$, UWT; (d) $b=0.9$, UHF. 

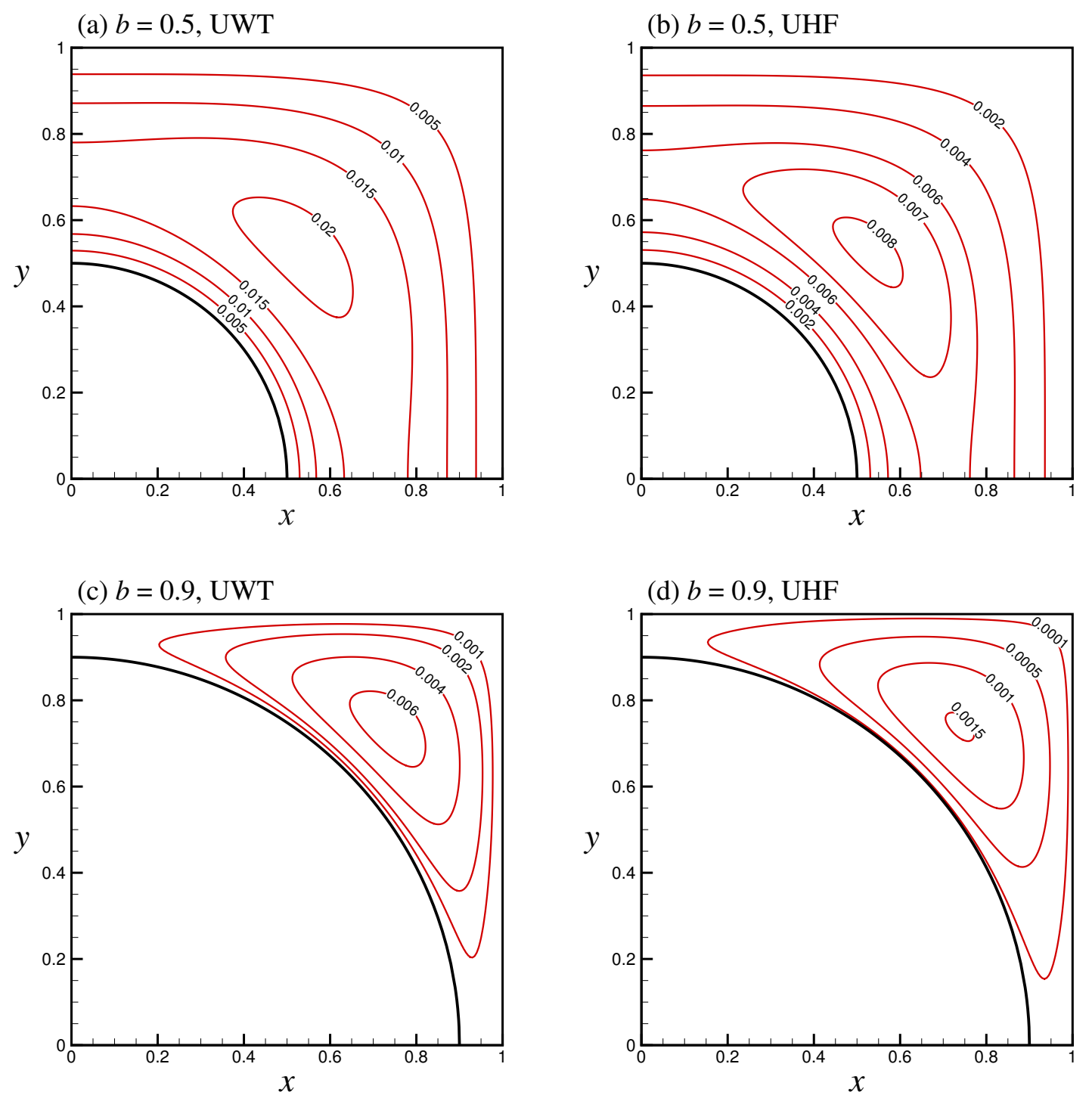

Figure 4: Velocity distribution $w(x, y)$ for $M=4$ (square), $\gamma=\lambda=0$ (i.e., no temperature jump and velocity slip), and (a) $b=0.5$, UWT; (b) $b=0.5$, UHF; (c) $b=0.9$, UWT; (d) $b=0.9, \mathrm{UHF}$. 

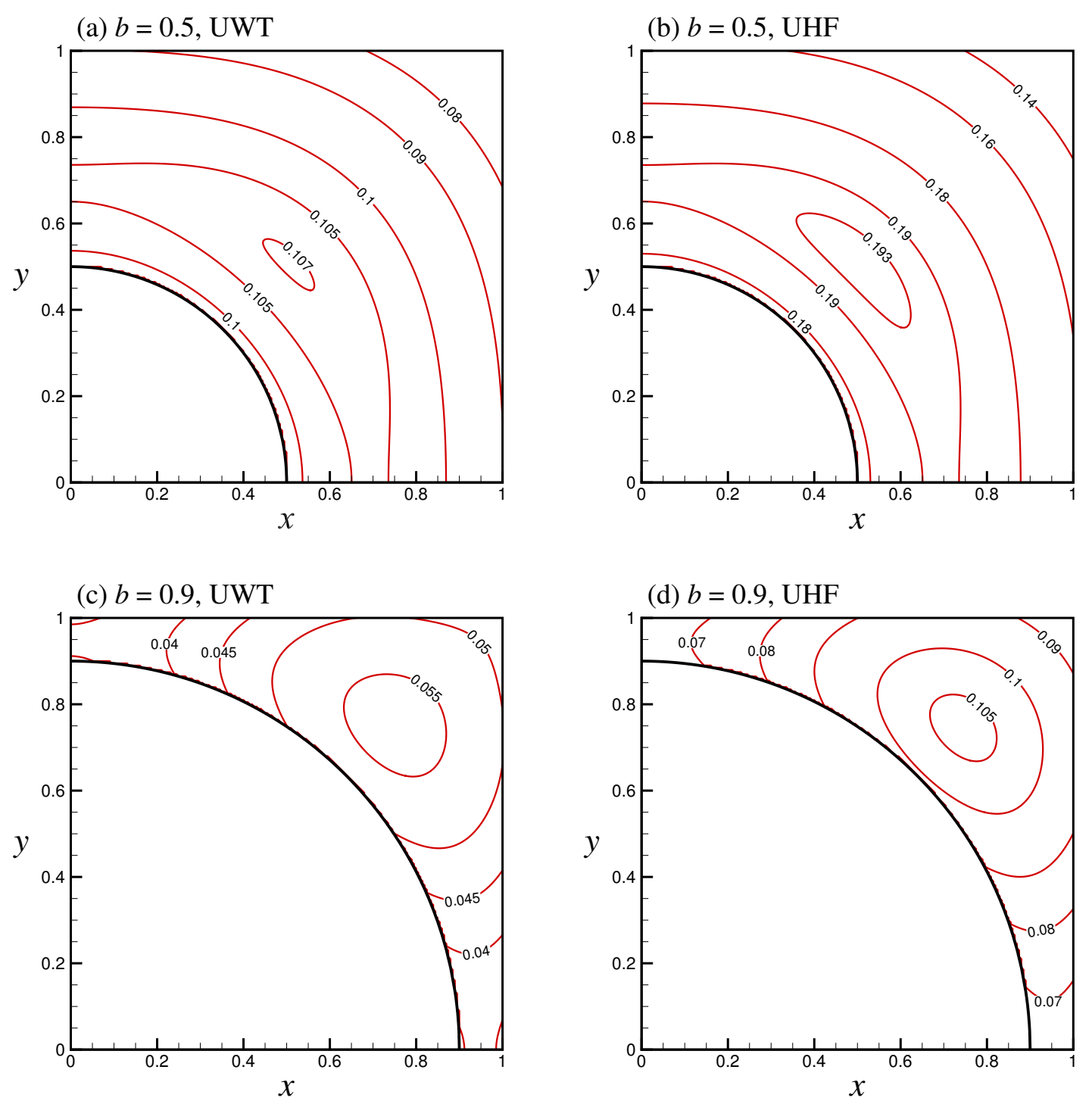

Figure 5: Velocity distribution $w(x, y)$ for $M=4$ (square), $\gamma=\lambda=1$ (i.e., finite temperature jump and velocity slip), and (a) $b=0.5$, UWT; (b) $b=0.5$, UHF; (c) $b=0.9$, UWT; (d) $b=0.9, \mathrm{UHF}$. 

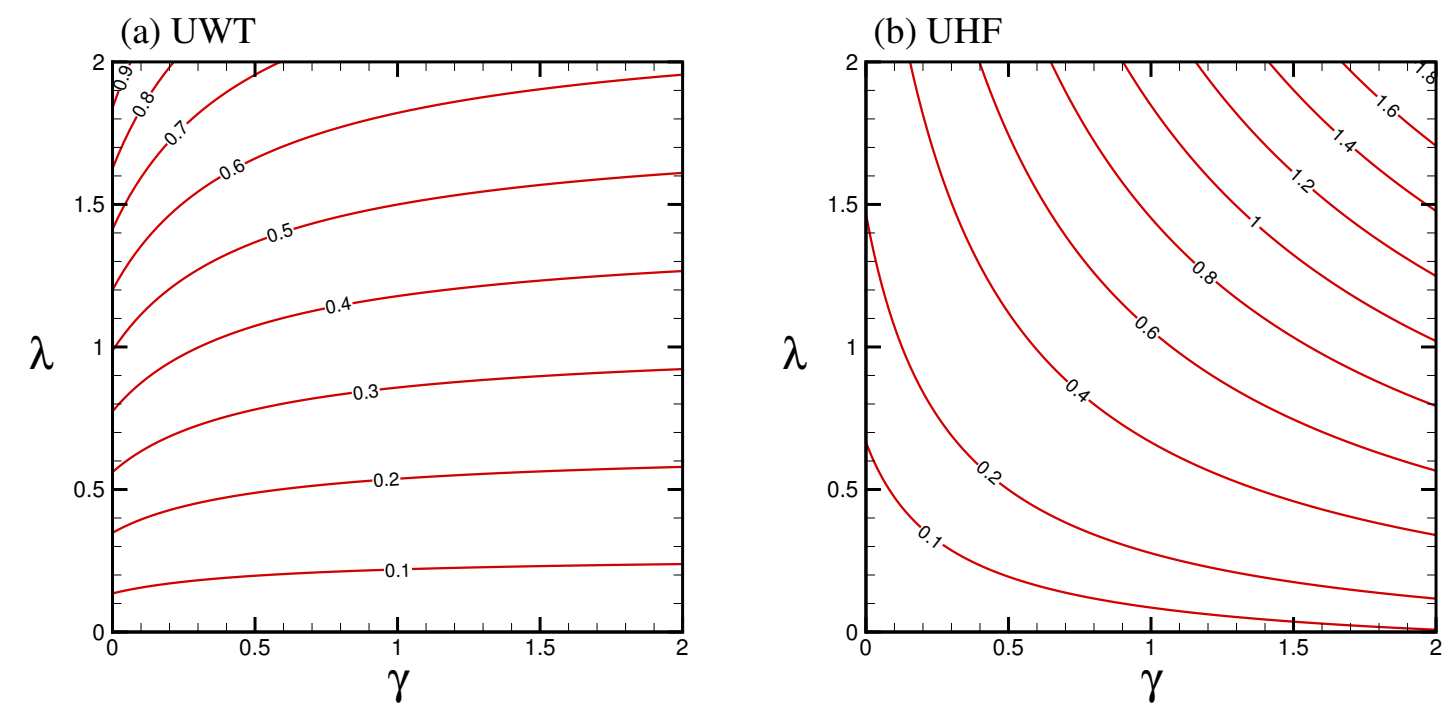

Figure 6: Flow rate $Q$ as a function of the temperature jump coefficient $\gamma$ and the slip length $\lambda$, for $M=4$ (square), $b=0.5$, and the core wall is at (a) UWT; (b) UHF. 
(a) UWT

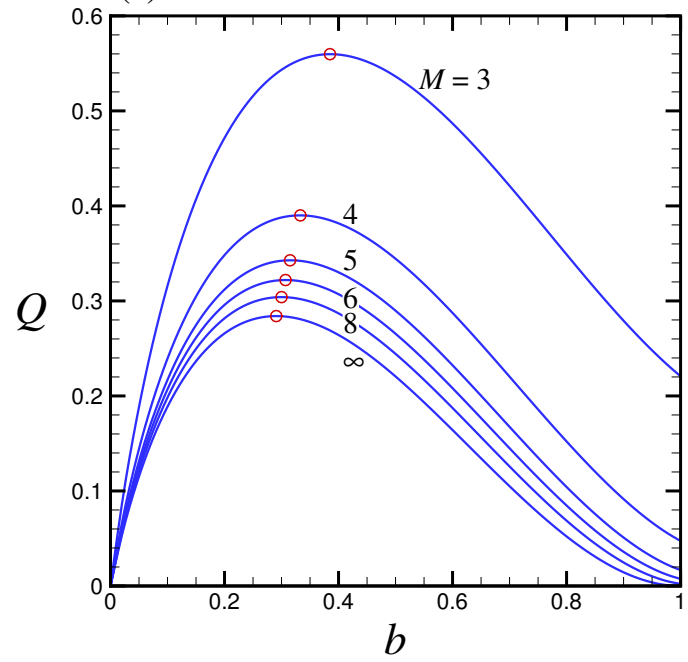

(b) UHF

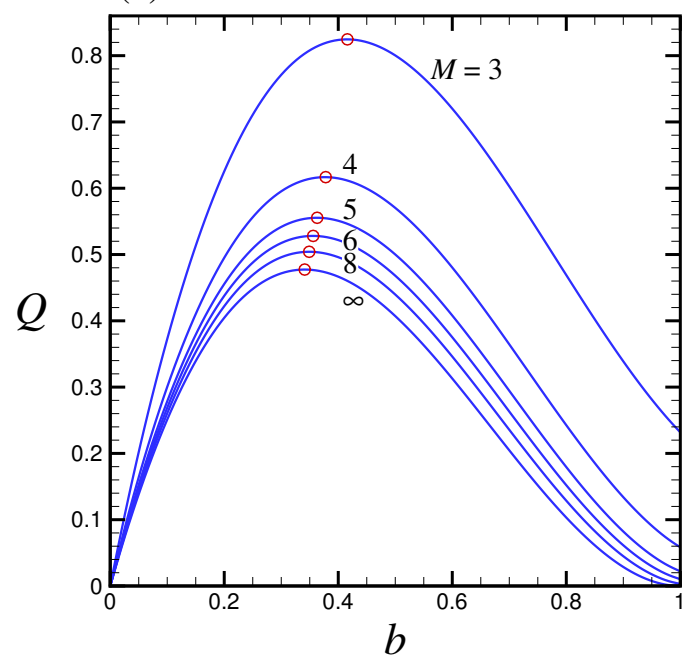

Figure 7: Flow rate $Q$ as a function of the core radius $b$, for $M=3,4,5,6,8, \infty, \gamma=\lambda=1$, and the core wall is at (a) UWT; (b) UHF. Values of the curve peaks, which are marked by the open circles, are given in Table 1. 
(a) UWT

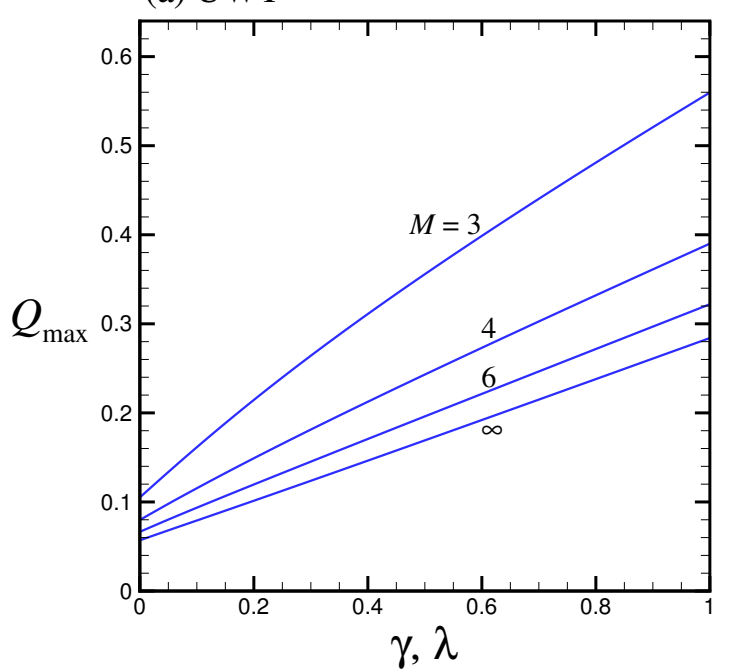

(b) UHF

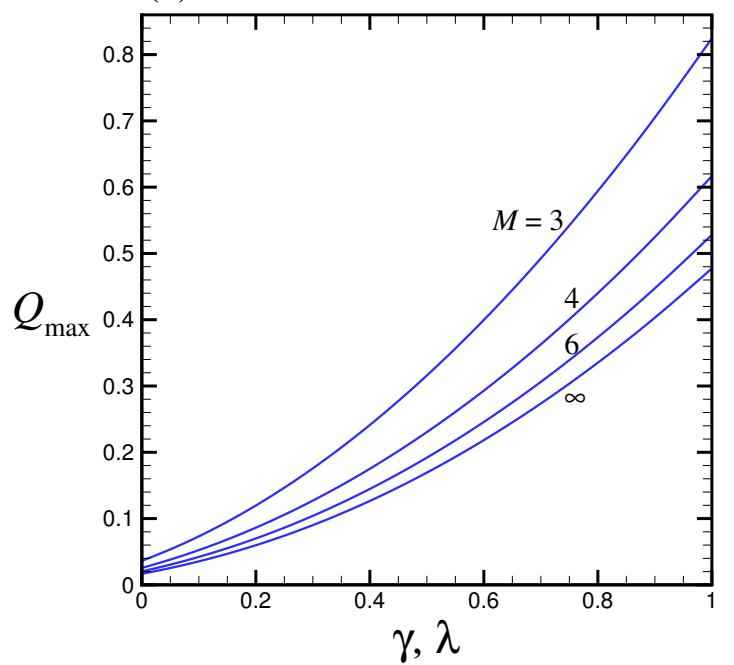

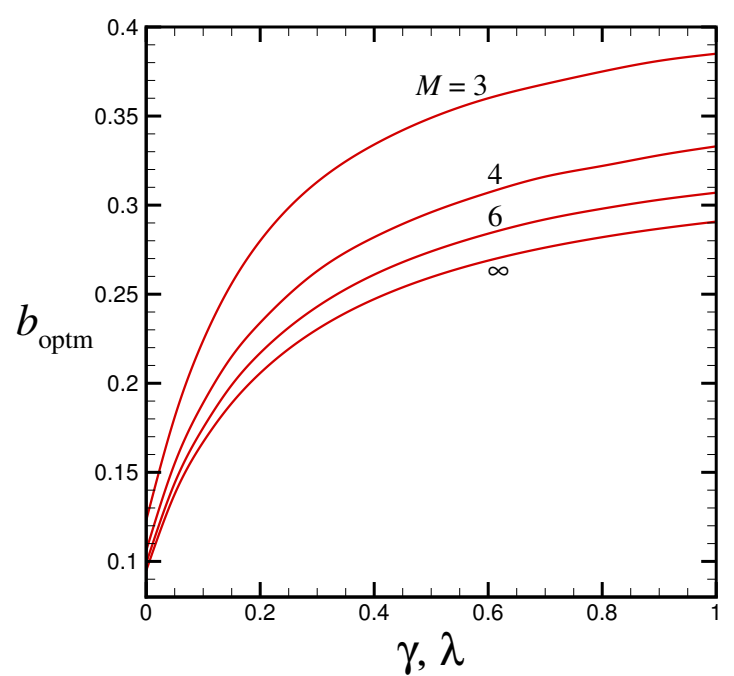

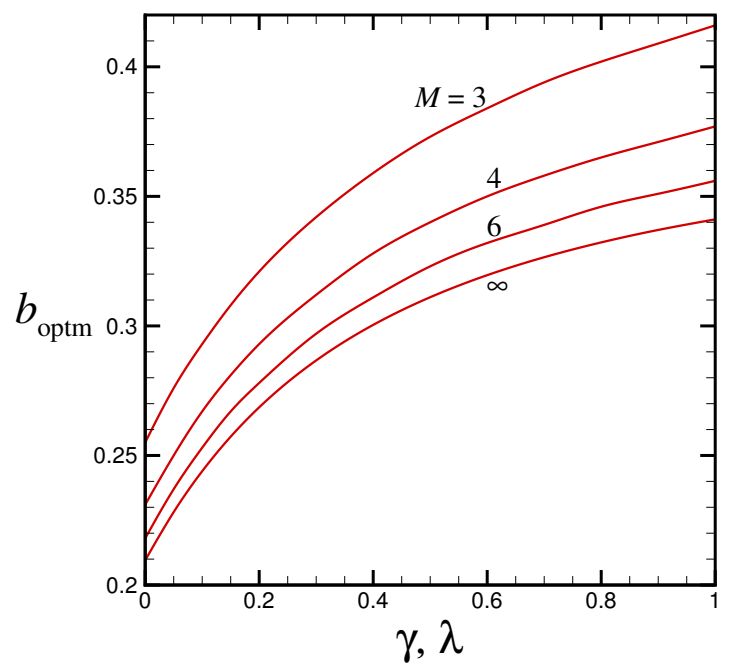

Figure 8: The maximum flow rate $Q_{\max }$ and the corresponding optimum core radius $b_{\text {optm }}$ as functions of the temperate jump coefficient $\gamma$ and the slip length $\lambda$, where $\gamma=\lambda$, for $M=3,4,6, \infty$, and the core wall at (a) UWT; (b) UHF. 